\title{
MORTALIDADE POR CAUSAS EXTERNAS NO MUNICÍPIO DE SÃO JOSÉ DO RIO PRETO, NO PERÍODO DE 1996-1998
}

\author{
INJURY DEATH IN SÃO JOSÉ DO RIO PRETO-1996-1998
}

Antonio M. M. Pires D’ Avila ${ }^{(1)}$; Augusto B. Oliveira ${ }^{(2)} \&$ Moisés Goldbaum $^{(3)}$

\begin{abstract}
(1)Médico Legista. Instituto Médico Legal do Estado de São Paulo. ${ }^{(2)}$ Docente. Departamento de Ciência de Computação e Estatística IBILCE-UNESP. ${ }^{\left({ }^{3}\right)}$ Docente. Departamento de Medicina Preventiva da Faculdade de Medicina - USP.

Correspondência: Antonio Miguel Morena Pires D’Ávila. Rua Siqueira Campos, no1590 - Bairro: Boa Vista,

CEP: 5025-055 - São José do Rio Preto- SP-Brasil. ampdavila@yahoo.com.br.
\end{abstract}

PIRES D' AVILA, A.M.M.; OLIVEIRA, A.B. \& GOLDBAUM, M. Mortalidade por causas externas no Município de São José do Rio Preto 1996-1998. Medicina, Ribeirão Preto37: 97-105, jan./jun. 2004.

RESUMO: Introdução: Um estudo epidemiológico sobre causas externas, utilizando taxas de mortalidade, foi realizado no Município de São José do Rio Preto, Estado de São Paulo, Brasil. O período considerado foi o triênio 1996-1998.

Objetivos: Descrever as taxas de mortalidade por causas externas no Município de São José do Rio Preto, no período de 1996-1998.

Metodologia: $O$ estudo foi baseado em dados oficiais do Sistema de Informação de Mortalidade do Ministério da Saúde, e de investigação baseada nas informações dos médicos legistas (IML).A análise dos dados foi feita através dos softwares Epinfo 6.0 e Tabwin.

Resultados: Os resultados foram comparados com os valores, obtidos na literatura, de algumas cidades e estados do Brasil. No caso de São José do Rio Preto, observou-se, no peírodo, diminuição no número dos óbitos por causas externas, particularmente nas mortes devidas a acidentes de trânsito, que são mais altas em relação aos valores observados no Brasil e no Estado de São Paulo. O estudo também detectou aumento no número absoluto de homicídios, principalmente nas faixas etárias de 15 a 39 anos de idade.

Conclusão: Os resultados demonstram a necessidade de iniciar programas de prevenção à mortalidade pelas citadas causas externas e monitorizar, em nível local, suas ocorrências .

UNITERMOS: Sistemas de Informação. Vigilância da População. Vigilância Epidemiológica. Medicina Preventiva; estatística \& dados numéricos. Prevenção de Acidentes. Coeficiente de Mortalidade.

\section{1- INTRODUÇÃO}

É consenso que, nos países mais desenvolvidos, ultrapassou-se a etapa em que os óbitos ocorriam, principalmente, por doenças infectocontagiosas, sendo que, nas últimas décadas, o problema maior tem sido localizado nas doenças cronicodegenerativas, neoplásicas e por causas externas, tornando-se, portanto, estas últimas, um importante e atual problema de Saúde Pública.
Pode-se conceituar agravo à saúde, lesão ou trauma por causas externas(CEX) qualquer dano intencional ou não, infringido ao corpo humano, como o resultado da exposição aguda à energia mecânica, térmica, elétrica ou química, ou pela ausência de elementos essenciais, como o calor ou o oxigênio. ${ }^{(1)}$

Uma vez conceituado o que se entende por causas externas, surge a necessidade de organizar a ocorrência de seus diversos tipos, de acordo com uma padronização, que é feita através da definição de caso, 
e que, também, permita a construção de uma classificação entre os mesmos. A oficialmente utilizada no nosso País é a divisão fornecida pela Organização Mundial de Saúde (OMS), que está inserida na Classificação Estatística Internacional de Doenças e Problemas Relacionados à Saúde (CID), no capítulo XX da $10^{\mathrm{a}}$ revisão, realizada no ano de 1989 . Devido à importância das causas externas, o que, nas revisões anteriores da CID, se constituída em uma classificação suplementar, recebeu, na atual, um capítulo específico, possibilitando a classificação de ocorrências e circunstâncias ambientais, como a causa de lesões, envenenamento e outros efeitos adversos. ${ }^{(2)}$

A CID utiliza, como critério de agrupamento de categorias, três caracteres relacionados à intencionalidade do agente causador da lesão, quais sejam: violência intencional- X60-Y09 (homicídio doloso) ou autoprovocada (suicida); acidental -V01-X59 (homicídio culposo) e quando ao classificador não for possível o enquadramento em uma das modalidades anteriores- Y10-Y98.

Nas últimas décadas, a violência tem se alastrado no meio urbano, por isso é importante monitorizar as comunidades de médio porte, que apresentam menores índices de homicídios, na tentativa de serem dedetectados precocemente, possíveis aumentos desses índices. Os dados obtidos através do monitoramento servirão como base para que se estabeleçam medidas adequadas ao enfrentamento dos problemas decorrentes ou geradores da violência.

O Município de São José do Rio Preto situa-se na Região Norte do Estado de São Paulo, possui área urbana de 83,46 Km2 e área total de 434,10 Km2. Sua população, em 1998, era de 340.010 habitantes.

Analisando-se o coeficiente da mortalidade (coeficiente por 100.000 habitantes) por causas externas, em residentes, e ocorrência no Município de São José do Rio Preto, no período de 1980-1999, observou-se que há um coeficiente, no mínimo, três vezes maior de acidentes em relação a homicídio e suicídio. Sistema de Informação de Mortalidade/Banco de Dados do Sistema Único de Saúde(SIM/DATASUS) Observou-se, ainda, que, no citado período, enquanto os coeficientes por homicídio e suicídio( por 100.000 habitantes) permanecem quase constantes, variando ente 0 e 10, o coeficiente por acidentes vem flutuando entre 30 a 70. Diante desses dados, sentiu-se que é necessário um entendimento mais abrangente da mortalidade por causas externas, no Município de São José do Rio Preto. Isso seria possível, através do estudo de coeficientes na população total, e para grupos específicos, por idade e sexo. Seria preciso, ainda, que os tipos de causas externas e suas circunstâncias fossem mais bem detalhados. Imbuídos de tal intuito, propôs-se este trabalho.

\section{2- OBJETIVOS}

\section{1- Objetivo Geral}

Descrever a mortalidade por causas externas na população residente, ocorrida no território do $\mathrm{Mu}$ nicípio de São José do Rio Preto, Estado de São Paulo- Brasil, no período de 1996 a 1998.

\section{2- Objetivos específicos}

Descrever a magnitude da mortalidade por causas externas, através de números absolutos e de seus coeficientes na população total e para grupos específicos por sexo e idade. Descrever a distribuição dos óbitos de acordo com cada um dos tipos de causas externas violência: homicídios e suicídios; acidentais: acidentes de trânsito e demais acidentes e outras causas externas.

\section{3- MATERIAL}

Para este estudo, dados secundários, de fontes oficiais, foram utilizados, tanto para as estimativas de população quanto para os óbitos e descritos a seguir.

\section{1- População}

Os dados de população foram estimados pela Fundação Sistema Estadual de Análise de Dados (SEADE) para o dia $1^{\circ}$ de julho de cada ano, a partir dos dados dos censos realizados pela Fundação Instituto Brasileiro de Geografia e Estatística(IBGE) nos anos abrangidos pelo estudo, e que nos foram cedidos através de planilhas fornecidas pela SEADE.

\section{2- Sistema de Informação de Mortalidade(SIM)}

Os dados oficiais de mortalidade tiveram como fonte as Declarações de Óbitos pertencentes a residentes, cuja ocorrência do evento fatal tenha sido no território do Município de São José do Rio Preto, e foram obtidos através de consulta nos arquivos do SIM do Ministério da Saúde(MS), fornecidos em discos compactos (CD) pela DATASUS/MS, abrangendo o período de 1980 a 1995; e pela Coordenadoria de Informática em Saúde/Secretaria de Estado da Saúde 
(CIS/SES) no período de $1^{\circ}$ de janeiro de 1996 a 31 de dezembro de 1998. Os dados foram ainda obtidos através de relatórios e de planilhas fornecidos pelo Núcleo de Informação (NI) da Divisão de Planejamento, Avaliação e Desenvolvimento(DPAD) da Direção Regional de Saúde XXII de São José do Rio Preto, da Secretaria de Estado da Saúde (SES/DIR/XXII).

\section{3- Instituto Médico Legal (IML)}

Os dados dos Laudos de Exame de Corpo de Delito, Necroscópicos (LECDN) foram obtidos através de consulta ao arquivo do setor de expediente e protocolo do Núcleo de Perícias Médico-Legais de São José do Rio Preto, do Instituto Médico-Legal do Estado, da Superintendência de Polícia Técnico-Científica da Secretaria de Segurança Pública do Estado de São Paulo. Os Laudos de Exame de Corpo de Delito foram os referentes aos óbitos de residentes, vítimas das CEX, e cuja ocorrência do evento fatal tenha sido no território do Município de São José do Rio Preto, no período de $1^{\circ}$ de janeiro de 1996 a 31 de dezembro de 1998.

\section{4-Variáveis e atributos estudados}

Em relação ao evento, conforme dados do SIM e dos LECDNs, as seguintes variáveis e atributos foram utilizados: sexo, idade e natureza da lesão.

\section{4- MÉTODO}

Trata-se de um estudo epidemiológico, descritivo.

Os dados foram apresentados segundo as variáveis estudadas em residentes do Município de São
José do Rio Preto e que tiveram a ocorrência do óbito no território deste município.

Em relação aos dados coletados no IML elaborou-se protocolo no qual se anotaram as variáveis e atributos de interesse ao estudo.

Para os cálculos dos coeficientes, foram utilizados, no denominador, dados de população, fornecidos pela SEADE, segundo sexo e idade estimada para $1^{\circ}$ de julho dos anos em estudo.

Os softwares Epinfo 6.0 e Tabwin foram utilizados para a análise dos dados.

\section{5- RESULTADOS}

De acordo com os dados fornecidos pela SEADE, e baseados no SIM e no IML, para os anos de 1996, 1997 e 1998, sobre os óbitos de residentes e ocorridos no Município de São José do Rio Preto, é que as causas de óbitos no presente estudo estão descritas.

Verificam-se na Tabela I, as principais causas de óbitos no Município de São José do Rio Preto e também que a mortalidade por CEX sofreu decréscimo no período 1996-1998.

Na Tabela II, observa-se, a ocorrência de óbitos por diferentes tipos de causas externas e o predomínio da mortalidade por acidentes de transporte bem como decréscimo da sua freqüência, no período estudado.

Verificou-se o predomínio de óbitos de indivíduos do sexo masculino e que a razão entre os dois sexos foi de 3,94 , quando tomados os anos em seu conjunto.

Tabela I: Óbitos em residentes e ocorridos no Município de São José do Rio Preto, segundo capítulo da CID 10, número absoluto e coeficiente por 100.000 habitantes- 1996-1998.

\begin{tabular}{|l|r|r|r|r|r|r|}
\hline Capítulo da CID 10 & $\mathbf{1 9 9 6}$ & Coef. & $\mathbf{1 9 9 7}$ & Coef. & $\mathbf{1 9 9 8}$ & Coef. \\
\hline I- Doenças Infecciosas e Parasitárias & 235 & 72,18 & 186 & 56,13 & 180 & 52,93 \\
\hline II- Neoplasias & 365 & 112,12 & 356 & 107,44 & 338 & 99,49 \\
\hline IX- Doenças Aparelho Circulatório & 598 & 183,69 & 652 & 196,78 & 616 & 181,17 \\
\hline X- Doenças Aparelho Respiratório & 211 & 64,81 & 272 & 82,09 & 279 & 82,05 \\
\hline XX- Causas Externas & 230 & 70,65 & 186 & 56,13 & 166 & 48,82 \\
\hline Total de Todos os Capítulos & 2.139 & 657,05 & 2.142 & 646,49 & 2.057 & 604,98 \\
\hline
\end{tabular}

Fonte: Seade/Datasus/Sim 


\begin{tabular}{|l|r|r|r|r|r|r|r|}
\hline $\begin{array}{l}\text { Tabela II: Óbitos em residentes e ocorridos no Município de São José do Rio Preto, segundo os subgrupos do } \\
\text { Capítulo XX da CIDBR-10 (CEX), ano, frequiência e coeficiente por 100.000 habitantes - 1996-1998 }\end{array}$ \\
\hline Capítulo XX subgrupo CIDBR-10 & $\mathbf{1 9 9 6}$ & Coef. & $\mathbf{1 9 9 7}$ & Coef. & $\mathbf{1 9 9 8}$ & Coef. \\
\hline 103. Acidentes de transporte & 101 & 31,02 & 81 & 24,44 & 60 & 17,64 \\
\hline 104. Quedas & 4 & 1,22 & 9 & 2,71 & 5 & 1,47 \\
\hline 105. Afogamento e submersão & 9 & 2,76 & 8 & 2,41 & 3 & 0,88 \\
\hline 106. Exposição ao fogo e fumaça & 2 & 0,61 & 3 & 0,90 & 1 & 0,29 \\
\hline 107. Envenenamento e intoxicação & 1 & 0,30 & - & 0,00 & 1 & 0,29 \\
\hline 108. Lesões autoprovocadas & 15 & 4,60 & 18 & 5,43 & 12 & 3,52 \\
\hline 109. Agressões & 28 & 8,60 & 30 & 9,05 & 34 & 9,99 \\
\hline 110. Eventos de intenção indeterminada & 17 & 5,22 & 10 & 3,01 & 10 & 2,94 \\
\hline 112. Todas as outras CEXs & 53 & 16,28 & 27 & 8,14 & 40 & 11,76 \\
\hline Capítulo XX-CEX Total
\end{tabular}

Fonte: SEADE/DATASUS/SIM

Na Tabela III, apresenta-se a mortalidade segundo o sexo e tipos de causas externas, observandose que ocorreu predomínio de óbitos por acidentes de transporte com pessoas de ambos os sexos, sendo maior com as do sexo masculino. Nota-se, ainda, um aumento, no número de óbitos por homicídios, com os indivíduos do sexo masculino, no mesmo período.

$\mathrm{Na}$ Tabela IV , ao agrupar-se a mortalidade por CEX, em três faixas etárias, utilizando-se coeficien- tes por 100.000 habitantes em cada uma das faixas, foram observados valores maiores na faixa etária de 50 ou mais anos, seguida pela de 15 a 49 anos, com exceção do ano de 1998, quando o total dos óbitos das faixas de 15 a 49 anos preponderaram. Os menores coeficientes foram observados na faixa de 0 a 14 anos de idade.

Ao analisar-se a mortalidade por acidente de transporte em relação ao sexo e às faixas etárias, veri-

Tabela III: Número de óbitos por causas externas CID 10(Capítulo XX), segundo o sexo, em residentes e ocorridos no Município de São José do Rio Preto- 1996-1998

\begin{tabular}{|c|c|c|c|c|c|c|c|c|c|}
\hline \multirow{2}{*}{$C E X$} & \multicolumn{3}{|c|}{1996} & \multicolumn{3}{|c|}{1997} & \multicolumn{3}{|c|}{1998} \\
\hline & $M$ & $\boldsymbol{F}$ & TOTAL & $M$ & $\boldsymbol{F}$ & TOTAL & $M$ & $\boldsymbol{F}$ & TOTAL \\
\hline Acidentes de Transporte & 81 & 20 & 101 & 63 & 18 & 81 & 53 & 7 & 60 \\
\hline Outros acidentes & 56 & 13 & 69 & 31 & 14 & 45 & 33 & 15 & 48 \\
\hline Suicídios & 13 & 2 & 15 & 14 & 4 & 18 & 9 & 3 & 12 \\
\hline Homicídios & 23 & 6 & 29 & 27 & 3 & 30 & 30 & 4 & 34 \\
\hline Demais CEXs & 13 & 3 & 18 & 12 & 2 & 14 & 9 & 3 & 12 \\
\hline TOTAL & 185 & 45 & 230 & 145 & 41 & 186 & 134 & 32 & 166 \\
\hline
\end{tabular}

Fonte: SEADE/DATASUS/SIM 
Tabela IV: Coeficiente da mortalidade por acidente de transporte, por 100.000 habitantes, segundo sexo e faixa etária em residentes e ocorridos no Município de São José do Rio Preto- 1996-1998

\begin{tabular}{|l|c|c|c|c|c|c|c|c|c|}
\hline & \multicolumn{3}{|c|}{$\mathbf{1 9 9 6}$} & & $\mathbf{1 9 9 7}$ & \multicolumn{3}{c|}{$\mathbf{1 9 9 8}$} \\
\hline Faixa Etária & $\boldsymbol{M}$ & $\boldsymbol{F}$ & $\mathbf{T O T A L}$ & $\boldsymbol{M}$ & $\boldsymbol{F}$ & $\mathbf{T O T A L}$ & $\boldsymbol{M}$ & $\boldsymbol{F}$ & TOTAL \\
\hline $0-14$ anos & 4,97 & 2,49 & 3,73 & 7,57 & 5,13 & 6,36 & 12,56 & - & 6,33 \\
\hline $15-49$ anos & 63,08 & 11,46 & 36,49 & 49,41 & 9,13 & 28,70 & 40,56 & 4,93 & 22,28 \\
\hline 50 ou + anos & 81,70 & 24,99 & 50,90 & 49,95 & 21,16 & 34,37 & 31,24 & 5,79 & 17,29 \\
\hline TOTAL & $\mathbf{5 1 , 4 3}$ & $\mathbf{1 1 , 4 3}$ & $\mathbf{3 1 , 0 2}$ & $\mathbf{3 9 , 1 9}$ & $\mathbf{1 0 , 5 5}$ & $\mathbf{2 4 , 4 4}$ & $\mathbf{3 2 , 1 1}$ & $\mathbf{4 , 0 0}$ & $\mathbf{1 7 , 6 4}$ \\
\hline
\end{tabular}

Fonte: SEADE/DATASUS/SIM

fica-se que ocorreu predominantemente com pessoas do sexo masculino e na faixa etária de 50 ou mais anos, nos anos de 1996 e 1997. No ano de 1998, houve predomínio de pessoas do sexo masculino, porém a faixa etária predominante foi a de 15 a 49 anos. Observa-se, ainda, que, no triênio ocorreu decréscimo no número de óbitos por acidentes de transporte.

Segundo os dados das vítimas de homicídio, que foram possíveis recuperar no IML-SJRP, calculou-se a idade média em 31,28 anos, com o desvio padrão de +/-14,74 anos; a variação etária oscilou entre 0 a 85 anos, tendo como mediana 29,5 anos; estando $25 \%$ dos casos até a idade de 21 anos; $75 \%$ dos casos até a idade de 39 anos, sendo a moda de 20 anos de idade.

Observou-se, também, que os homicídios sofreram aumento no período. Tomando por base o ano de 1996, houve 17,2\% de crescimento entre 1998/1996 e 13,3\% entre 1998/1997, sendo a razão de mortalidade masculina de 3,9, 9,5 e 7,9 nos anos de 1996, 1997 e 1998, respectivamente.

Nas vítimas de suicídio, a idade média foi de 41 anos, com desvio padrão de $+/-18$ anos; variando a idade de 17 a 86 anos; tendo, como mediana, 37 anos; $25 \%$ dos casos ocorreram até a idade de 28 anos e $75 \%$ dos casos até a idade de 53 anos, sendo a moda 30 anos de idade.

Nas vítimas fatais de acidentes de transporte, a idade média foi de 37,2 anos, com desvio padrão de+/ -20,62 anos, tendo amplitude de variação etária entre 0 e 94 anos, apresentando, como mediana, 33 anos, estando $25 \%$ dos casos até a idade de 22 anos, $75 \%$ dos casos até a idade de 50 anos, sendo a moda de 20 anos de idade.

Observa-se, na Tabela V, pelos dados recuperados dos Laudos de Exame de Corpo de Delito Necroscópico do IML/SJRP, que o traumatismo
cranioencefálico(TCE) representou $45 \%$ dos óbitos no período. Quando estudadas apenas as lesões por acidentes de transporte, o impacto na cabeça(TCE), seja do ocupante do veículo, seja do pedestre, alcançou a cifra de 57\% dos casos. Quanto aos homicídios, em $64,9 \%$ dos casos, foi relatada a existência de hemorragia, tendo, como instrumento de lesão, a arma de fogo ou a arma branca. A mesma análise, feita para os suicídios, mostrou predominância do traumatismo cranioencefálico também em 33\% das vítimas deste tipo de CEX.

\section{6- DISCUSSÃO}

Nos países mais desenvolvidos, nas últimas décadas, a mortalidade devida a doenças infectocontagiosas tem declinado, enquanto se tem observado um aumento da mortalidade devida a doenças não transmissíveis ou cronicodegenerativas e neoplásicas, nas camadas mais idosas da população, e as chamadas causas externas que atingem os mais jovens. $\mathrm{O}$ fenômeno foi chamado de Transição Epidemiológica. ${ }^{(3)} \mathrm{A}$ situação, no Brasil, não é homogênea, varia de região para região, e a mortalidade por causas externas tem se tornado um importante e atual problema de Saúde Pública.

Atualmente, nos países do Primeiro Mundo, as mortes violentas são devidas, principalmente, a acidentes de trânsito e suicídios. ${ }^{(4,5,6)}$ Nos EUA, os homicídios, especialmente os envolvendo adultos e jovens de raça negra, superam os suicídios, diferindo, assim, dos demais países desenvolvidos. ${ }^{(7,8)}$

No Brasil, os dados de mortalidade indicam um crescimento insidioso da violência, sobretudo nos grandes centros urbanos, que concentram $75 \%$ das ocorrências. As CEX eram responsáveis por $2 \%$ no con- 
Tabela V: Número de óbitos por causas externas, segundo a natureza da lesão (CID-10), em residentes e ocorridos no Município de São José do Rio Preto-1996-1998

\begin{tabular}{|c|c|c|c|c|c|c|}
\hline$C I D$ & $\begin{array}{c}\text { Acidentes de } \\
\text { Transporte }\end{array}$ & Suicídios & Homicídios & Outras & Total Cex & Causa Básica \\
\hline R58 & 17 & 4 & 61 & 10 & 92 & Hemorragia Traumática \\
\hline R59 & 0 & 0 & 0 & 1 & 1 & Paralisia Bulbar \\
\hline R79 & 3 & 0 & 0 & 0 & 3 & Choque Hipovolêmico \\
\hline R99 & 1 & 1 & 1 & 7 & 10 & Indeterminada \\
\hline S02 & 13 & 2 & 1 & 4 & 20 & $\begin{array}{l}\text { Fratura e Afundamento do } \\
\text { Crânio }\end{array}$ \\
\hline S06 & 153 & 13 & 23 & 41 & 230 & Traumatismo Cranioencefálico \\
\hline S19 & 1 & 0 & 0 & 0 & 1 & Degolamento \\
\hline $\mathrm{T} 03$ & 1 & 0 & 0 & 1 & 2 & Traumatismo Raquimedular \\
\hline T06 & 6 & 0 & 0 & 2 & 8 & Traumatismo Toracoabdominal \\
\hline T07 & 35 & 2 & 2 & 9 & 48 & Politraumatismo \\
\hline T09 & 3 & 0 & 0 & 1 & 4 & Traumatismo Medular-Cervical \\
\hline $\mathrm{T} 31$ & 0 & 0 & 1 & 11 & 12 & Intoxicação Endógena \\
\hline $\mathrm{T} 65$ & 0 & 7 & 0 & 2 & 9 & Intoxicação Exógena Aguda \\
\hline $\mathrm{T} 71$ & 0 & 2 & 1 & 2 & 5 & Asfixia Mecânica Externa \\
\hline $\mathrm{T} 75$ & 0 & 0 & 0 & 1 & 1 & Anóxia Anóxica (afogado) \\
\hline $\mathrm{T} 79$ & 32 & 1 & 1 & 4 & 38 & Choque por Traumatismo \\
\hline T91 & 1 & 0 & 0 & 0 & 1 & $\begin{array}{l}\text { Complicação Tardia } \\
\text { (peritonite) }\end{array}$ \\
\hline$X 70$ & 0 & 7 & 2 & 16 & 25 & Asfixia Mecânica \\
\hline Y87 & 1 & 0 & 1 & 1 & 3 & Septicemia \\
\hline TOTAL & 267 & 39 & 94 & 113 & 513 & \\
\hline
\end{tabular}

Fonte: IML-SJRP

junto da mortalidade, em geral, em 1930, e em 1980 e em 1993, saltaram para $10,5 \%$ e $15 \%$ respectivamente. ${ }^{(9)}$

Drumond, estudando as causas de mortalidade na população de 15 a 74 anos de idade, ressaltou um aumento dos riscos de mortes, especialmente no sexo masculino, por doenças crônicas não transmissíveis, como câncer, doenças cardiovasculares e violência. ${ }^{(10)}$

Os principais registros de morte por causa externa, no País, ainda são os de acidentes de trânsito, que apresentam coeficientes e tendências diferenciados em diversos Estados da Federação e suas capitais. ${ }^{(11)}$ Estudos realizados, nas cidades do Rio de Janeiro e de São Paulo, mostraram que é com a população adulta que se concentra a maior parcela de óbitos por acidentes de trânsito. ${ }^{(12,13)}$

Reichenhein \& Werneck concluíram que os acidentes de trânsito, no Estado e no Município do Rio de Janeiro, em 1990, foram a terceira causa de anos 
de vida potencialmente perdidos entre 1 e 70 anos. Na cidade de São Paulo, em 1991, segundo dados da Prefeitura Municipal (1992) foram a quinta causa. Nos últimos anos, em ambas as cidades, tais indicadores foram ultrapassados pelos homicídios. ${ }^{(14)}$

Na última década do século XX, os coeficientes de mortalidade por causas externas, principalmente por acidentes de trânsito e homicídios, são crescentes em todas as faixas etárias e com pessoas dos dois sexos, destacando-se, no entanto, entre adultos jovens, do sexo masculino, em áreas urbanas habitadas por populações em piores condições socioeconômicas, fruto da iniqüidade a que esta parcela de nossa população vem sendo submetida, principalmente nas regiões metropolitanas de São Paulo, Rio de Janeiro, Recife e Vitória. ${ }^{(13,15 / 21)}$

Estudo comparativo entre a mortalidade por CEX, nas cidades do $\mathrm{ABCD}$, Região Metropolitana da Grande São Paulo, nos anos de 1970-1992, mostrou aumento acentuado dos coeficientes, exceto para São Bernardo do Campo, que mostrou descenso. Em relação ao sexo, $80 \%$ dos indivíduos eram do sexo masculino. Os coeficientes maiores foram encontrados entre as faixas etárias de 9 a 39 anos. Aproximadamente $80 \%$ das vítimas eram solteiras ${ }^{(22)}$.

Vasconcelos Filho estudou a ocorrência de homicídios na cidade de Fortaleza, no período de 1987-1996, e encontrou predomínio com pessoas do sexo masculino (9:1) e coeficientes maiores na faixa etária entre 20 e $49 \operatorname{anos}^{(23)}$.

Mello Jorge, fazendo uma análise baseada no SIM, no período de 1980 a 1995, por sexo, grupo etário e causa de morte, encontrou as CEX (acidentes e violência) em lugar de destaque, representando, em todas as regiões, de 60 a $80 \%$ das mortes com pessoas do sexo masculino e de 20 a $50 \%$ com as de sexo feminino). ${ }^{(24)}$

Os resultados de algumas análises mostram, particularmente nas grandes cidades brasileiras, que os homicídios estão basicamente constituídos por conflitos com a Polícia, desavenças entre grupos de narcotraficantes e desentendimentos interpessoais, e que os homicídios e os acidentes de trânsito, juntos, representam mais de 50\% das mortes por CEX. Do ponto de vista sociodemográfico, a grande maioria das vítimas são adolescentes e adultos jovens do sexo masculino, com características típicas das camadas menos favorecidas da população, residentes em áreas pobres e, às vezes, periféricas das grandes metrópoles, de cor negra, com baixa escolaridade e pouca ou nenhuma qualificação profissional. ${ }^{(25)}$
Observa-se, em números absolutos, que $90 \%$ dos homicídios ocorrem nas áreas urbanas ${ }^{(21)}$, lembrando-se que a concentração de habitantes nessas áreas suplanta a concentração de habitantes das áreas rurais do País.

Especificamente em relação aos homicídios ocorridos no Estado de São Paulo, dados apresentados no Caderno Segurança, elaborado pela SEADE para o Fórum São Paulo Século XXI, mostram que, no ano de 1998, foram registrados, por 100.000 habitantes, 48,4 homicídios na Capital e 19,6 no Interior. Em relação às lesões corporais dolosas, registraram-se, por 100.000 habitantes, 297,0 ocorrências na Capital e 634,5 no Interior, o que traz, intuitivamente, a tradução de que os conflitos diversos resultam em morte na Capital, e em agressões físicas, no Interior. ${ }^{(26)}$

As mudanças que ocorreram nos últimos quarenta anos no Brasil, mais especificamente no Estado de São Paulo, em relação à mortalidade por CEX, acompanharam as mudanças no meio-ambiente e também no estilo de vida das pessoas, ou seja, em dois parâmetros básicos, associados às causas de mortalidade. No período, houve maior migração da industrialização para o Interior do Estado, grande desenvolvimento da indústria automobilística e a expansão da rede rodoviária estadual; também, aproximação maior entre os estilos de vida das pessoas das cidades do Interior e as da Capital, baseados na economia de consumo, inserida em um regime capitalista.

O perfil da mortalidade por CEX, em relação aos homicídios, acompanhou o processo de industrialização e de urbanização sem planejamento no Brasil, sendo que é similar na periferia das metrópoles, e, nas décadas mencionadas, seguindo o deslocamento do parque industrial, no Estado de São Paulo, para as cidades do eixo São Paulo- São José dos Campos- Rio de Janeiro e São Paulo- Campinas- Ribeirão Preto.

Primeiramente, foi observado, através das séries históricas, que a frequiência de óbitos por acidentes de trânsito, que era o maior problema nas cidades do Interior Paulista, passou a ser substituída pela de homicídios, ao redor desses eixos de deslocamento industrial.

Observa-se que as cidades paulistas entre 300.000 e 500.000 habitantes, encontram-se ao redor da Capital ou estão posicionadas nos eixos acima apontados. O Município de São José do Rio Preto apresenta-se fora dos citados eixos e, também, com as menores taxas de homicídios dentre as cidades de seu porte, no Estado de São Paulo. Por outro lado, o suicídio 
não representa posição de destaque no obituário por CEX, e, através dos relatórios periódicos da SEADE, observa-se que, nos locais onde ocorrem mais homicídios, a sua freqüência é mais baixa e vice-versa.

Quando agrupados os 582 óbitos ocorridos no triênio 1996-1998, em São José do Rio Preto, pôde-se observar que, tomando-se por base o ano de 1996, houve um decréscimo nos valores de coeficientes para os acidentes de transporte e um crescimento para os homicídios. Os demais agrupamentos, quer intencionais (suicídios) ou demais causas acidentais, apesar de sofrerem flutuações, apresentaram-se, no ano de 1998, em níveis menores, com exceção das quedas.
Diante do crescimento expressivo da violência em todos os setores da sociedade, o monitoramento constante dos coeficientes e índices de mortalidade por CEX, em municípios de médio porte, como o de São José do Rio Preto, é de fundamental importância no sentido de acompanhar tais causas externas no tempo, pois as variações são indicadores da qualidade de vida das comunidades. Baseando-se no estudo desses indicadores, podem ser implementados programas de prevenção à escalada de tais eventos fatais. Está claro que é mais fácil manter a saúde da coletividade do que tentar recuperá-la, até porque ainda não se chegou a um consenso sobre as verdadeiras raízes da violência.

PIRES D' AVILA, AMM; OLIVEIRA, AB \& GOLDBAUM, M Injury death in São José do Rio Preto 1996-1998. Medicina, Ribeirão Preto 37: 97-105, jan./june 2004.

ABSTRACT: Introduction:An epidemiological study of the mortality rates by injuries in São José do Rio Preto, State of São Paulo, Brazil, has been carried out. The period considered is 1996-1998.

Objectives: To show injuries mortality rates in São José do Rio Preto-SP in 1996-98.

Methods: The study was based on official data from the Mortality Information Service of the Brazilian Ministry of Health, and in a survey based on data from medical forensic examiners (IML).Software Epinfo6.0 and Tabwin was utilized for data analises.

Results:The results were compared to values obtained in the literature for the other cities of São Paulo and Brazil. In the case of São José do Rio Preto, was observed a decrease in the number of deaths associated with external causes in the period, but particularly in deaths due to traffic accidents with motor vehicles an increase in cases of pedestrians killed by hit-and-run drivers, that is much larger than the corresponding values for Brazil and for the State of São Paulo. The study showed increase in rates of homicides, mainly in the 15 to 39 years age group .

Conclusion:This result suggests that is important to start programs to reduce the number of deaths by injuries, as well as the necessity of monitoring locally all these data.

UNITERMS: Information Systems. Population Surveillance. Epidemiologic Surveillance. Preventive Medicine; statistic \& numerical data. Accident Prevention. Mortality Rate.

\section{REFERÊNCIAS BIBLIOGRÁFICAS}

1 - INJURY CONTROL. Washington, DC: National Academy Press. Am J Prev Med 5, 1989. Supl.103.

2 -ORGANIZAÇÃO MUNDIAL DE SAÚDE (OMS). Classificação estatística internacional de doenças e problemas relacionados à saúde, Revisão de 1989, 10ª ed., EDUSP, São Paulo, v.1, 1997.

3 - LAURENTI R. Transição epidemiológica. Apresentado na Mesa Redonda A questão demográfica e a transição epidemiológica, Congresso Brasileiro de Epidemiologia, Campinas, 1990.

4 - PANEQUE RJ. La mortalidad del adulto de 15 a 49 anos em Cuba. Níveles y evolución, 1960-1982. Rev Cuba Adm Salud 12:339-349, 1986.
5 - WIGLE D T; MAO Y; SEMENCIW R; MC CANN C \& DAVIS J W. Premature deaths in Canada: impact, trends and opportunities for prevention. Can J Public Health 81: 376-381, 1990.

6 - HANSLUWKA H. Some aspects of adult mortality in developed countries. Cah Sociol Demograf Med 31: 170-176, 1989.

7 - HOMICIDE AMONG YOUNG BLACK MALES- UNITED STATES, 1978-1987. Morb Mortal Wkly Rev 39: 869-873, 1990.

8 - MORTALITY TRENDS AND LEADING CAUSES OF DEATH AMONG ADOLESCENTS AND YOUNG ADULTS-UNITED STATES, 1979-1988. Morb Mortal Wkly Rev 42: 459-461, 1993.

10 - DRUMOND Jr. M. Mortalidade evitável do adulto: Desigualdades sócio-espacias no município de São Paulo. Dissertação de Mestrado, Faculdade de Ciências Médicas da Universidade Estadual de Campinas, Campinas, p.15-26, 1996. 
11 - MELLO JORGE MHP \& LATORRE MRDO. Acidentes de trânsito no Brasil; Dados e tendências. Cad Saúde Pública 10: 19-44, 1994.

12. WILKINSON RG. Unhealthy societies:from inequality to well being, Routledge, London, p 63-76,1997.

13 - GAWRYSZEWSKI WP. A mortalidade por causas externas no município de São Paulo, Dissertação de Mestrado, Faculdade de Saúde Pública da USP, São Paulo, p1-136, 1995.

14 - REICHENHEIN ME \& WERNECK GL. Anos potenciais de vida perdidos no Rio de Janeiro, As mortes violentas em questão. Cad Saúde Pública 10 :188-198, 1994.

15 - MELLO JORGE MHP. Mortalidade por causas violentas no Município de São Paulo. Tese de Doutoramento, Faculdade de Saúde Pública da USP, São Paulo, p1-72, 1979.

16 - SOUZA ER.\& MINAYO MCS. O impacto da violência social na saúde pública do Brasil: década de 80. In MINAYO MCS, org. Os muitos Brasis: Saúde e população na década de 80, Hucitec, São Paulo, p. 97-115,1995.

17 - MINAYO MC \& SOUZA ER. O limite da exclusão social: Meninos e meninas de rua no Brasil. Hucitec/ Abrasco, São Paulo/Rio de Janeiro, p 32-44,1993.

18 - VEIGA JA. O perfil de saúde dos trabalhadores urbanos, baseados na mortalidade de população, entre 15 e 64 anos de idade, residentes nas regiões metropolitanas brasileiras, 1979-1988. Dissertação de Mestrado, Faculdade de Saúde Pública da USP, São Paulo, p.1-189, 1995.

19 - ZALUAR A \& ALVITO M. Um século de favela. Editora. Fundação Getúlio Vargas, Rio de Janeiro, p 52-63, 1998.
20 - LIMA RS. Conflitos sociais e criminalidade urbana; uma análise dos homicídios cometidos no município de São Paulo, São Paulo. Dissertação de Mestrado, Faculdade de Filosofia, Letras e Ciências Humanas da USP, São Paulo, $p$ $1-126,2000$

21 - POMPEU JC. Levantamentos de registros de homicídios no Brasil (1979-98). (Relatório da Secretaria Nacional de Segurança Pública). Ministério da Justiça, Brasília, 2000. (Comunicação pessoal)

22 - GALVANESE GCSM. Mortalidade por causas externas nos municípios de Santo André, São Bernardo, São Caetano do Sul e Diadema (São Paulo) de 1979 a 1992. Dissertação de Mestrado, Faculdade de Saúde Pública da USP, São Paulo, p.1-213,1998.

23 - VASCONCELOS F. Homicídios na cidade de Fortaleza: O espaço de criação e desenvolvimento na vida em sociedade, Dissertação de Mestrado, Universidade Federal do Ceará, Fortaleza, p 27-42, 1998.

24 - MELLO JORGE MHP. Como morrem nossos jovens. In Jovens acontecendo na trilha das políticas públicas. Comissão Nacional de População e Desenvolvimento, Brasília, p 41-56,1998

25 - MINAYO MC \& SOUZA ER. É possível prevenir a violência? Reflexos a partir do Campo da saúde pública. Ciência \& Saúde Coletiva, ABRASCO, Rio de Janeiro, 4: 7-23, 1999.

26 - ASSEMBLÉIA LEGISLATIVA/FSEAD. Fórum São Paulo Século XXI. Caderno de Segurança, DOE. IMESP, São Paulo, 2000

Recebido para publicação em 18/08/2003

Aprovado para publicação em 23/04/2004 